\title{
Ethological study of flower visiting insects in two parks, Kolkata, India
}

\author{
Subhodeep Sarkar ${ }^{1}$, Abantika Nandy ${ }^{2}$, Soumendra Nath Talapatra ${ }^{2, *}$ \\ ${ }^{1}$ Department of Environmental Science, Asutosh College \\ S. P. Mukherjee Road, Kolkata, India \\ *Phone: 91-33-2461-5445 \\ ${ }^{2}$ Department of Environmental Science, University of Calcutta \\ 51/1 \& 2 Hazra Road, Kolkata 700019, India \\ *E-mail address: ecologylive@yahoo.co.in
}

\begin{abstract}
Flower visiting insects attract by the flowers colour, shape, size and fragrance as pollinator. This is a mutual relationship between flowers and insects. The present study aims to know behavioural response or ethology of flower visiting insect in relation to particular flower and/or probable atmospheric changes in two managed parks, Kolkata, India. The study areas were selected as per heavily-populated neighborhoods, nearby office buildings, nearby roads and continuous vehicular movements, human interactions as visitors. The study was carried out at 2 sampling stations viz (i) Elliot park and (ii) Agri-Horticulture Society. The flower species were selected viz. Helianthus annuus (sunflower), Petunia sp. (petunia) and Buganvilia spectabilis (Buganvilia) planting above mentioned areas because these species are more common among other species. In each flower, behavioural response or ethology of visiting insects were studied by visual observation and total 10 flowers of each species were observed randomly. The present results clearly indicate that various insects were majorly showed foraging and feeding behaviour and only mating behaviour was found in two species. This study is a preliminary assessment of flower visiting insects' ethology but further researches are needed in relation to pollination efficiencies of flower visiting insects in the particular flower and air pollution load nearby area by using instruments. It was concluded that insect visitors are showing foraging and feeding behavior but only two species were showed mating behaviour, which may be due to the vehicular air pollution because two parks are located nearby roads and continuous vehicular movements were observed.
\end{abstract}

Keywords: Insect behavior; Vehicular pollution; Flower visiting insects; Insect ethology

\section{INTRODUCTION}

Behavioral study or ethology of animal is the study of adaptive behavior in relation to social and environmental circumstances and the natural selection shapes behavior have been reported by Bird and O'Connell (2006). It deals with the ways in which behavior may be adaptive by allowing an animal to increase or even maximize its reproductive success. The branch of ecology as ethology examines the adaptive significance of behavior, or how behavior may increase survival and reproduction in species. In case of insects, there was 
majorly observed searching or foraging behavior. Besides this, three different kinds of interaction have been observed between flower and insect: (1) pollinating behavior, (2) feeding behavior, (3) mating behavior. Pollination is one of the most fascinating aspects of interaction between flowering plant (s) and insect (s). Generally the extent of interdependence is regulated by phenology, floral characters as well as by form, structure and behavior of the pollinators. During evolution flowers have developed various strategized for attracting insects (Ram and Mathur, 1984).

Earliest terrestrial insects likely were scavengers may have initially used plants by feeding directly on leaves, roots, spores or pollen. Plant feeding really takes off, when flowering plants develop. There are some categories of feeding interaction between flowering plant and insect. These are (1) leaf chewer (eat entire leaves or large portions of leaves), (2) plant miner and borers (mining involves burrowing into leafs or just below the surface of stems, fruits or roots, boring involves burrowing more deeply into stems, roots, or fruits), (3) sap sucker (pierce plant tissue to obtain liquid from either phloem or xylem), (4) seed predator (some eat the entire seed, but many feed on the inside of the seed by boring into it), (5) nectar or pollen feeder and (6) gall former (galls are an abnormal or pathological growth in plants, done as a response to some invader).

Some insect individuals carried visible pollen loads, females seemed to spend more time on flowers than males, and male spent greater proportion of their time searching for mates (Schlinger, 1956) and the mating takes place within the flower patches. (Borkent and Schlinger, 2008).

Matteson et al. (2012) have documented the presence of herbaceous flowering plants and flower-visiting insects (e.g. Diptera, Coleoptera, Lepidoptera, Hymenoptera) across a wide range of urban green spaces' and residential and commercial blocks. According to Bell (1990), searching behavior is an active movement by which insects seek resources viz. food, mates, oviposition and nesting sites and refugia. It is an important kind of behavior, was already observed in the insects because these resources are absolutely essential for their growth, development, and maintenance in an individual and for ensuring the success of future generations. The searching mechanisms are efficient and accurate assessment mechanisms in which it can be determined the crucial part for an individual's chances of survival and reproduction. It was also documented the searching behavior incurred costs in addition to the energy used for locomotion. Insects visit flowers for several reasons, the predominant reason being for food (Alamu et al., 2013). The mating behavior of insect species is a fundamental issue because it helps to know the conservation of important species or destruction of pest species. The mating behavior is a very complex phenomenon involving several factors (Murvosh et al., 1964). It was also reported that insects of various groups visit flowers in search of mates (Proctor et al., 1996; Sugiura, 2007).

Many studies have already been carried out in discrete urban habitats (e.g. city parks, meadow remnants, community and private gardens) by many research have affirmed the importance of floral resources for floral-feeding insects such as bees and other arthropods (Ram and Mathur, 1984; Cane 2005; Hernandez et al. 2009; Matteson et al., 2012, Alamu et al., 2013). However, few studies have documented across the spectrum of urban habitats, including residential, commercial, and different types of green spaces, to enable an evaluation of the effects of land use heterogeneity on biotic communities within cities (Hennig and Ghazoul 2011; Sattler et al. 2010; Wojcik 2011; Chowdhury et al., 2014). Chowdury et al., (2014) have studied the diversity of flower visiting insects in managed park but no one has attempted before the ethological aspects flower visiting insects in the managed park. 
The present study aims to know behavioural response or ethology of flower visiting insect in relation to particular flower in two managed parks, Kolkata, India.

\section{MATERIALS AND METHODS}

The study was carried out at 2 sampling stations viz (i) Elliot park at Jwaharlal Nehuru road, (latitude $=22^{\circ} 32^{\prime} \mathrm{N}$ and longitude $=88^{\circ} 20^{\prime} \mathrm{E}$ ) and (ii) Agri-horticulture Society at Alipore Road (latitude $=22^{\circ} 31^{\prime} \mathrm{N}$ and longitude $=88^{\circ} 19^{\prime} \mathrm{E}$ ), situated at 5.18 meters above sea level. The study was carried in dry season.

The flower species were selected viz. Helianthus annuus, Petunia sp. and Buganvilia $s p$. growing near roadside of above mentioned areas because these species are more common among other species. These plant species produce flowers at different times during the year and thus, they are good sources of pollen and nectar to the different types of insects that forage on flowers. The direct and indirect influences on flower visiting insects in two urban habitats, ethological assessment was done on specific floral and insect distributions by transects randomly within these two parks and observed the insect pollinators on the basis of foraging, feeding and mating behavior.

The sampling design was involved identifying insects onto the specific flowering species with the help of literatures, books, museum specimens, internet etc. and also by visual identification. Behavioural study was done by visual observation, image capture, etc. All the activities of insects during the visit onto flower were recorded and tabulated in Table 1. These two sampling stations mainly two parks were selected on the basis of human interference, moderate and heavy traffic density and continuous vehicular movement nearby roads as per visualization.

\section{RESULTS}

In the present study, the behavioral responses or ethology of flower visiting insects were observed in two managed parks, namely Elliot park and Agri-Horticulture Society. In these two parks, the flower visiting insects ethological observation was made on three particular flowering species viz. Petunia sp., Helianthus annuus and Bougainvillea spectabilis, which were very common in these two parks. The ethology of insects on these flowering species, 10 species of individual flowering species were randomly observed and identified individual insect species and their behavioural response were tabulated in Table 1. In comparison with Agri-horticulture society, various insects viz. small red and black ant, fire ant, dragon fly, lady bug beetle, honeybee, large black ant, bumble bee, hoverfly were observed in Elliot park while in Agri-horticulture society, it was observed some of the above mentioned species alongwith psyche butterfly, leaf beetle, blue/green bottle fly, stick insect and crab spider.

In the present ethological study, it was observed several flower visiting insects and they have showed behavioural respons onto the flower. These insect species viz. the small red and black ants were found in Petunia sp. Bougainvillea sp. in Elliot park, and was absent in sunflower and was mainly showed nectar searching behavior and crawling behavior on the petal. But in Agri-horticulture society, ant was present only in sunflower and absent in other flowers and showed the same activity like Elliot Park. Dragonfly was very less in number in Elliot Park than Agri-horticulture society. 
Table 1. Flower visiting insect behavior or ethological study between two parks.

\begin{tabular}{|c|c|c|c|c|c|c|c|}
\hline \multirow{3}{*}{ SI No. } & \multirow{3}{*}{$\begin{array}{l}\text { Flower } \\
\text { species }\end{array}$} & \multicolumn{3}{|c|}{ ELLIOT PARK } & \multicolumn{3}{|c|}{ AGRI-HORTICULTURE SOCIETY } \\
\hline & & \multirow[b]{2}{*}{$\begin{array}{c}\text { Insect } \\
\text { species }\end{array}$} & \multicolumn{2}{|c|}{ Behavioral response } & \multirow[b]{2}{*}{$\begin{array}{l}\text { Insect } \\
\text { species }\end{array}$} & \multicolumn{2}{|c|}{$\begin{array}{l}\text { Behavioral } \\
\text { response }\end{array}$} \\
\hline & & & $\begin{array}{l}\text { Foraging and } \\
\text { Feeding } \\
\text { behavior }\end{array}$ & $\begin{array}{c}\text { Mating } \\
\text { behavior }\end{array}$ & & $\begin{array}{c}\text { Foraging and } \\
\text { Feeding } \\
\text { behavior }\end{array}$ & $\begin{array}{l}\text { Mating } \\
\text { behavior }\end{array}$ \\
\hline \multirow[b]{2}{*}{1.} & & 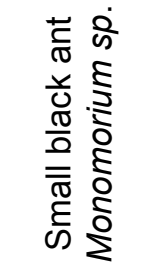 & 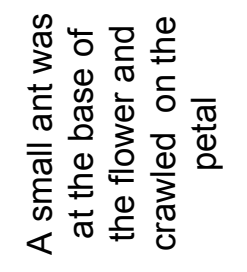 & 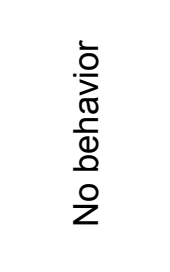 & \multirow{2}{*}{ 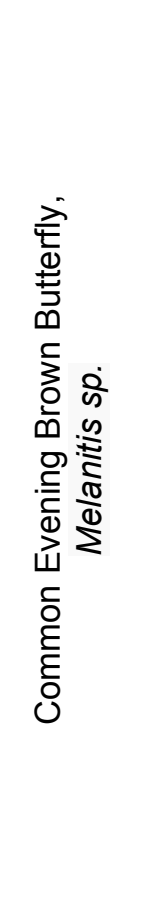 } & \multirow{2}{*}{ 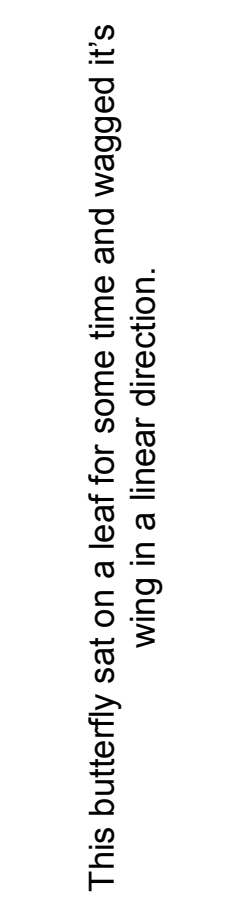 } & \multirow[b]{2}{*}{ 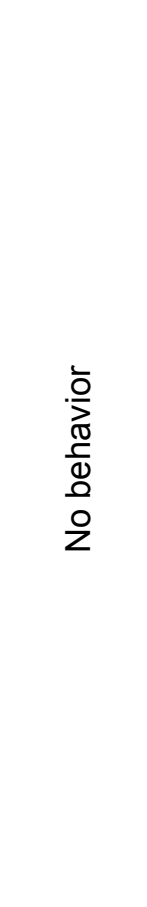 } \\
\hline & $\begin{array}{l}0 \\
0 \\
0 \\
\frac{0}{2} \\
\mathbb{2} \\
0 \\
0\end{array}$ & 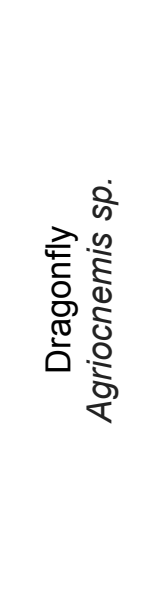 & 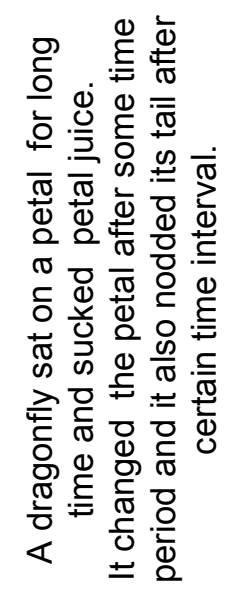 & 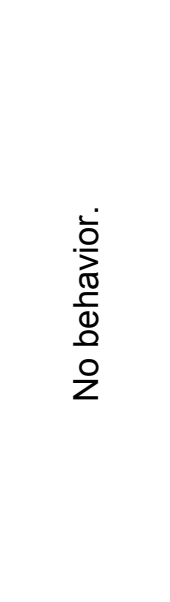 & & & \\
\hline 2. & 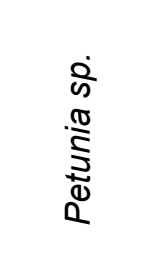 & 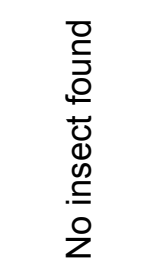 & 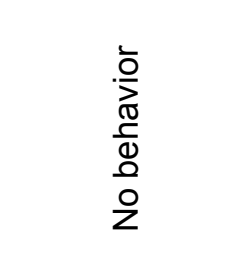 & 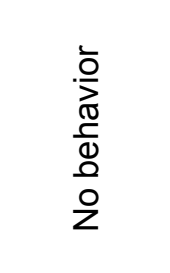 & 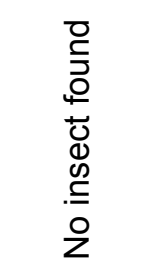 & 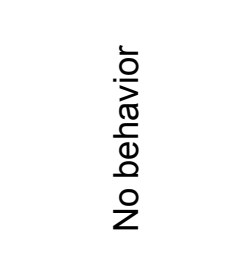 & 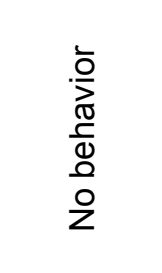 \\
\hline 3. & 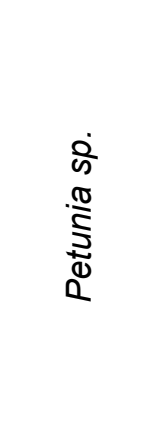 & 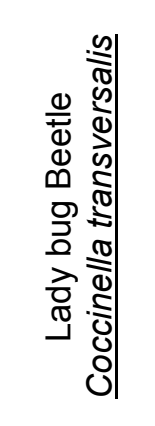 & 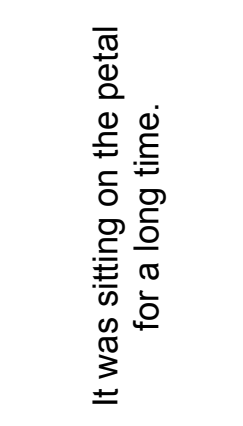 & 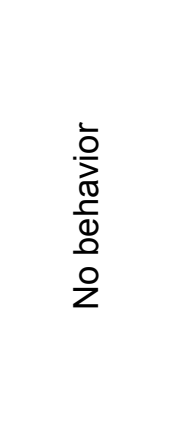 & 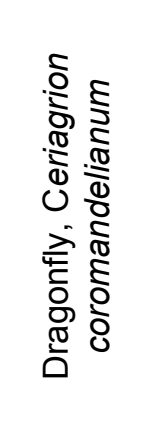 & 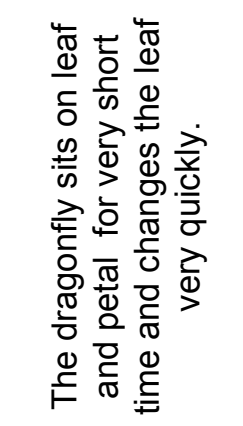 & 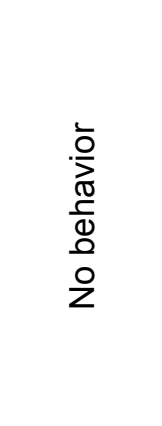 \\
\hline
\end{tabular}




\begin{tabular}{|c|c|c|c|c|c|c|c|}
\hline 4. & 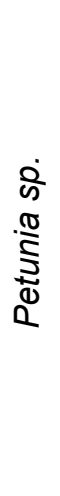 & 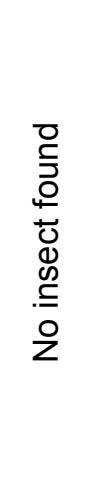 & 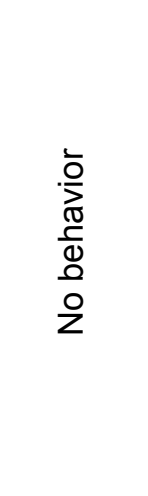 & 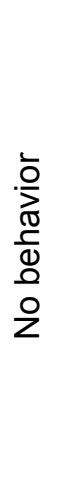 & 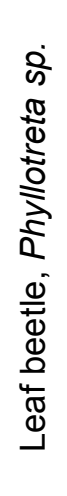 & 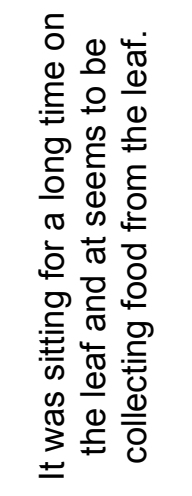 & 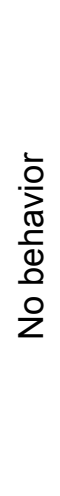 \\
\hline 5. & 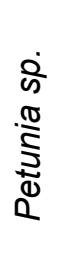 & 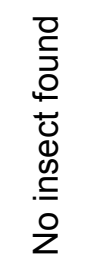 & 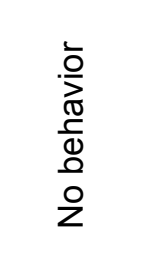 & 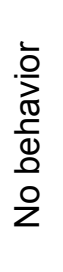 & 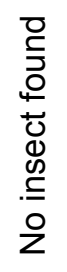 & 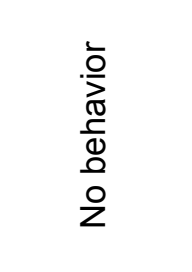 & 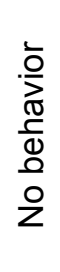 \\
\hline 6. & 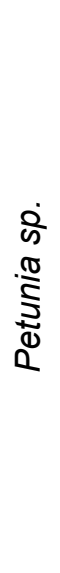 & 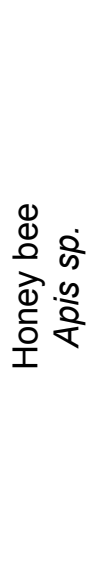 & 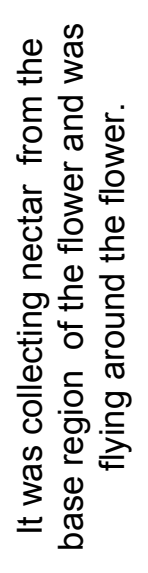 & 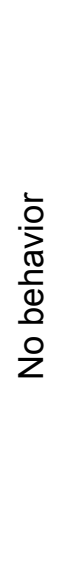 & 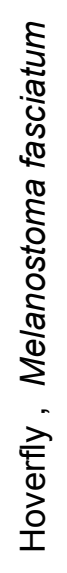 & 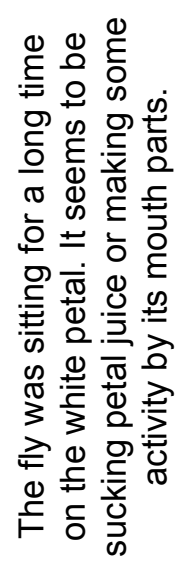 & 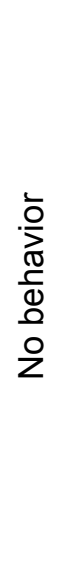 \\
\hline 7. & 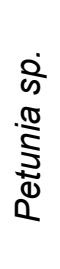 & 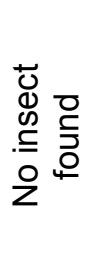 & $\begin{array}{l}\frac{\overline{0}}{2} \\
\frac{0}{0} \\
\frac{0}{0} \\
0 \\
0 \\
z\end{array}$ & 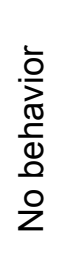 & 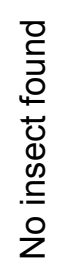 & 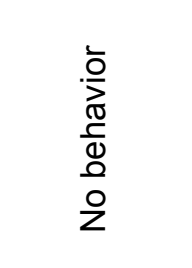 & 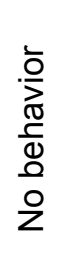 \\
\hline 8. & $\begin{array}{l}\frac{\dot{0}}{4} \\
\frac{0}{0} \\
\frac{0}{3} \\
0 \\
0\end{array}$ & 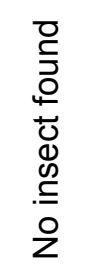 & 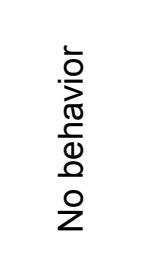 & 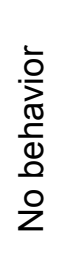 & 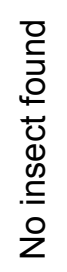 & 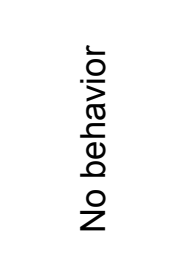 & 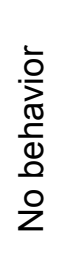 \\
\hline
\end{tabular}




\begin{tabular}{|c|c|c|c|c|c|c|c|}
\hline 9. & $\begin{array}{l}\frac{1}{0} \\
\text { D. } \\
\frac{\pi}{5} \\
\frac{1}{0} \\
0 \\
0\end{array}$ & 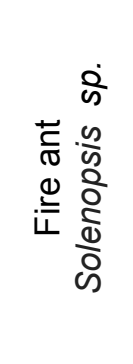 & 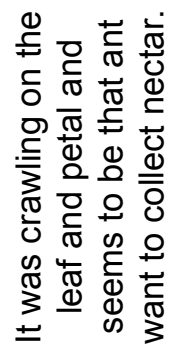 & $\begin{array}{l}\frac{.}{2} \\
\frac{\pi}{d} \\
\frac{1}{d} \\
\frac{0}{0} \\
\frac{2}{z}\end{array}$ & 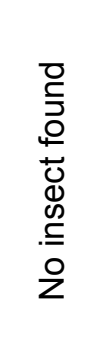 & 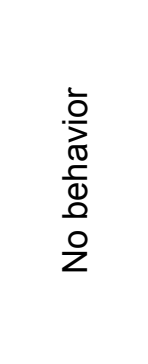 & $\begin{array}{l}\frac{1}{2} \\
\frac{0}{2} \\
\frac{0}{0} \\
0 \\
0 \\
\frac{0}{Z}\end{array}$ \\
\hline 10. & $\begin{array}{l}\dot{0} \\
\dot{0} \\
\frac{\pi}{0} \\
\stackrel{5}{1} \\
0 \\
0\end{array}$ & 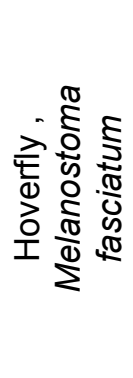 & 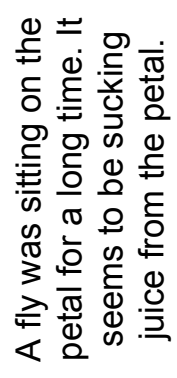 & 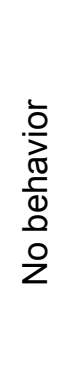 & 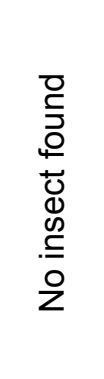 & 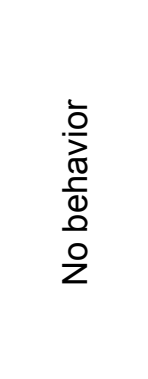 & 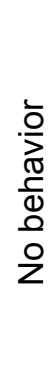 \\
\hline \multirow{2}{*}{11.} & \multirow{2}{*}{ 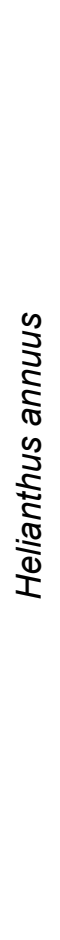 } & \multirow{2}{*}{ 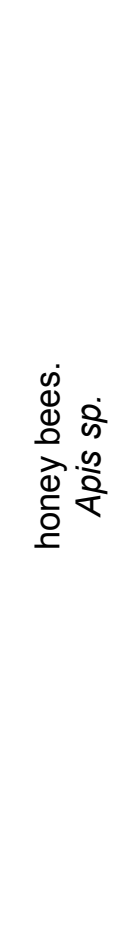 } & \multirow{2}{*}{ 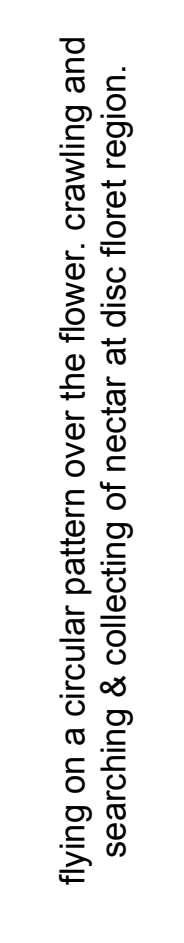 } & \multirow{2}{*}{$\begin{array}{l}\frac{.}{2} \\
\frac{\pi}{0} \\
\frac{1}{0} \\
0 \\
0 \\
z\end{array}$} & 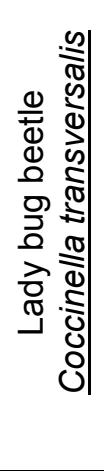 & 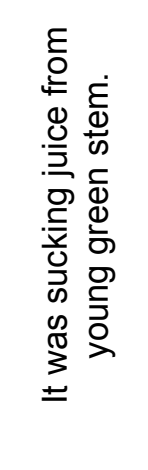 & $\begin{array}{l}\grave{o} \\
\frac{0}{2} \\
\frac{\pi}{0} \\
0 \\
0 \\
\frac{0}{2}\end{array}$ \\
\hline & & & & & 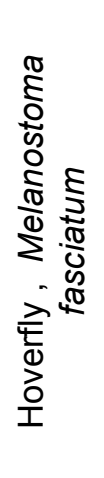 & 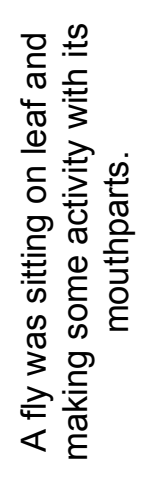 & $\begin{array}{l}\grave{o} \\
\frac{0}{d} \\
\frac{0}{0} \\
0 \\
0 \\
\frac{0}{2}\end{array}$ \\
\hline 12. & 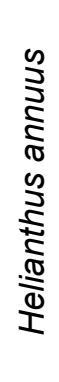 & 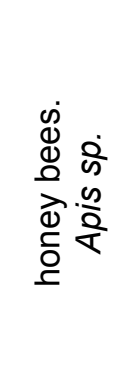 & 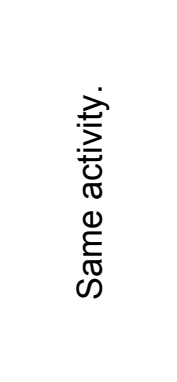 & 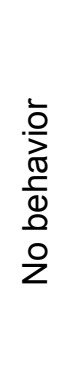 & 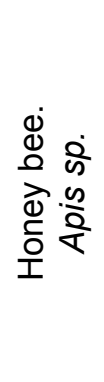 & 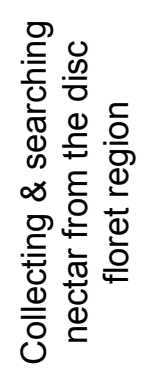 & $\begin{array}{l}\grave{o} \\
\frac{0}{d} \\
\frac{0}{d} \\
0 \\
0 \\
2\end{array}$ \\
\hline
\end{tabular}




\begin{tabular}{|c|c|c|c|c|c|c|c|}
\hline 13. & 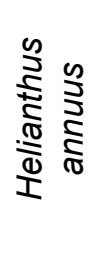 & 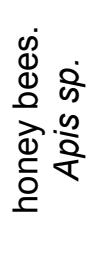 & 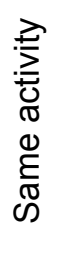 & $\begin{array}{l}\frac{0}{2} \\
\frac{0}{0} \\
\frac{0}{0} \\
0 \\
\frac{0}{2}\end{array}$ & 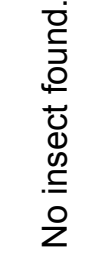 & 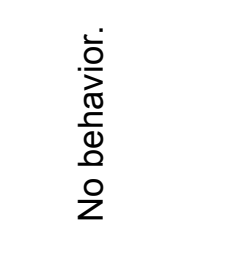 & 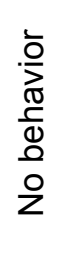 \\
\hline 14. & 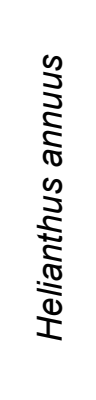 & 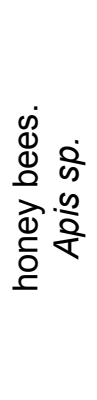 & 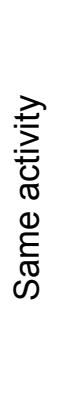 & 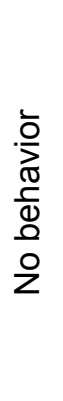 & 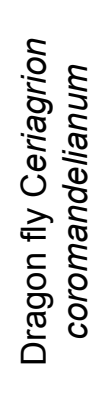 & 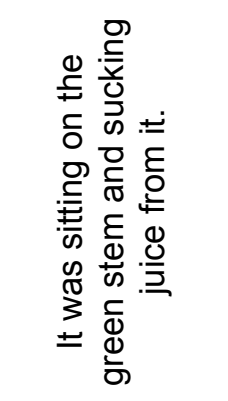 & 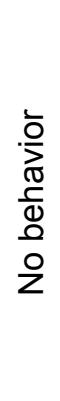 \\
\hline 15. & 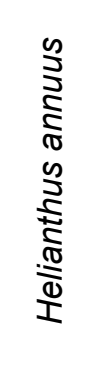 & 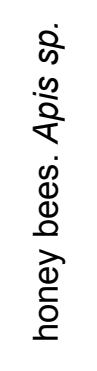 & 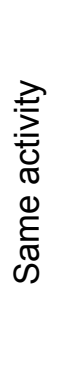 & 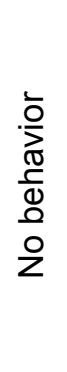 & 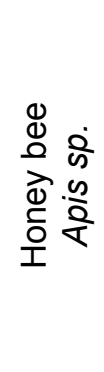 & 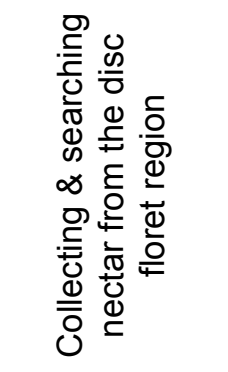 & 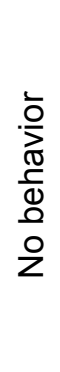 \\
\hline 16. & 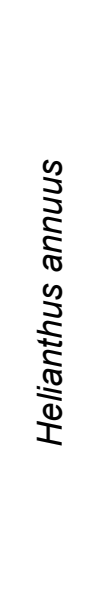 & 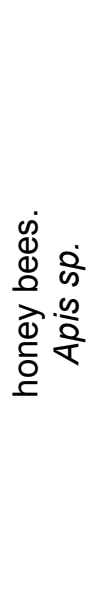 & 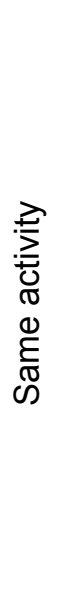 & $\begin{array}{l}\frac{0}{2} \\
\frac{0}{0} \\
\frac{0}{0} \\
0 \\
\text { ¿ }\end{array}$ & 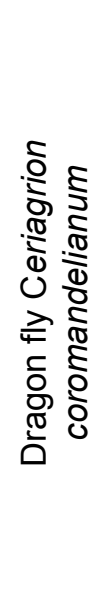 & 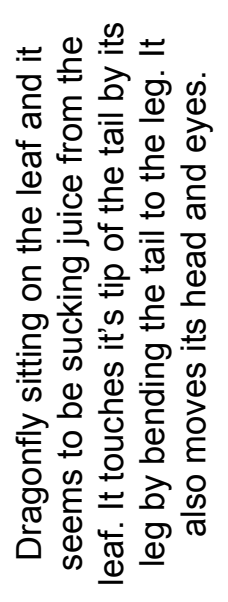 & 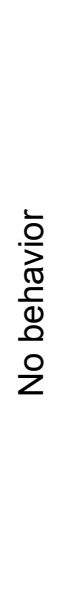 \\
\hline 17. & 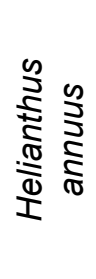 & 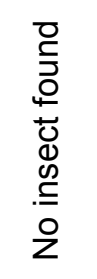 & 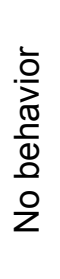 & 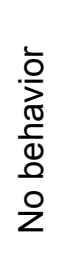 & 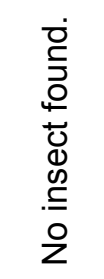 & 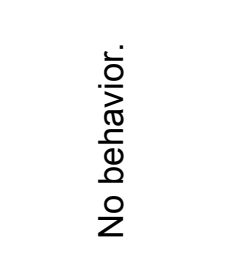 & 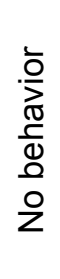 \\
\hline
\end{tabular}




\begin{tabular}{|c|c|c|c|c|c|c|c|}
\hline 18. & 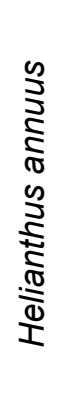 & 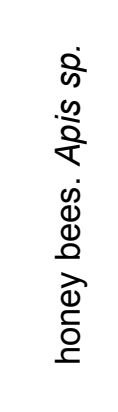 & 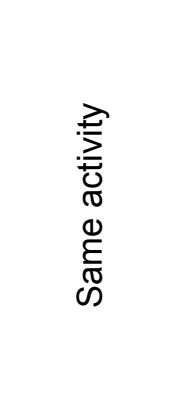 & 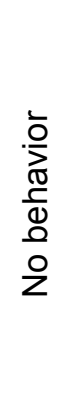 & 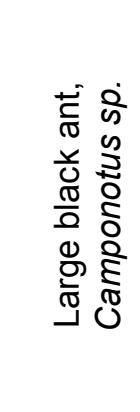 & 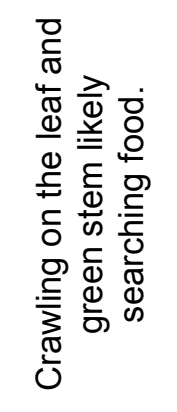 & $\begin{array}{l}\overline{0} \\
\frac{0}{\pi} \\
\frac{\pi}{0} \\
0 \\
0 \\
0 \\
z\end{array}$ \\
\hline 19. & 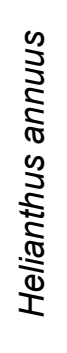 & 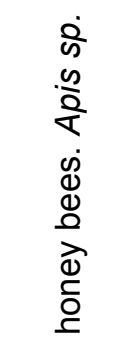 & 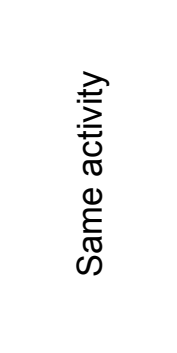 & 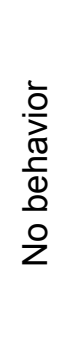 & 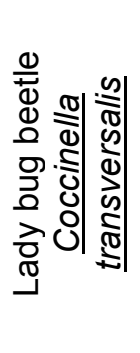 & 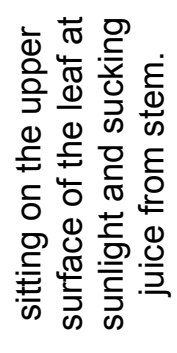 & 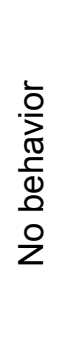 \\
\hline \multirow[b]{2}{*}{20.} & \multirow{2}{*}{ 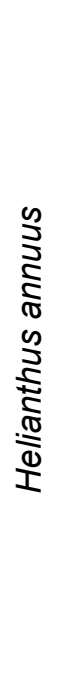 } & \multirow[b]{2}{*}{ 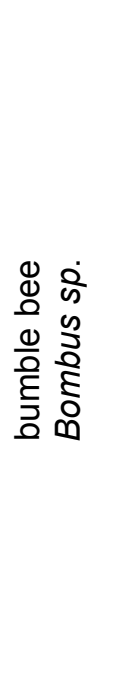 } & \multirow{2}{*}{ 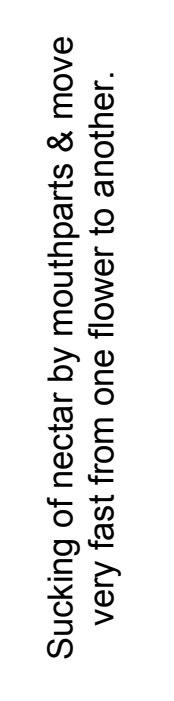 } & \multirow[b]{2}{*}{ 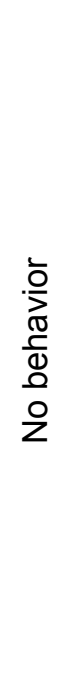 } & 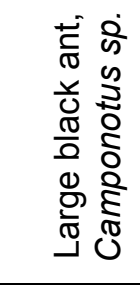 & 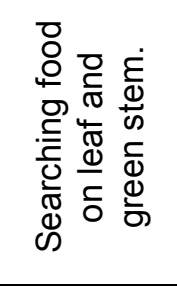 & 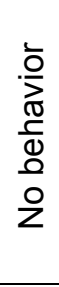 \\
\hline & & & & & 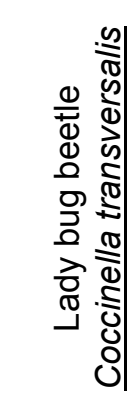 & 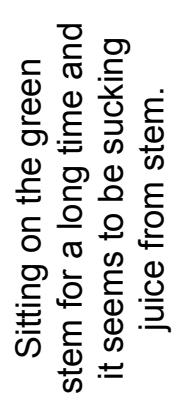 & 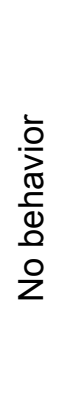 \\
\hline 21. & 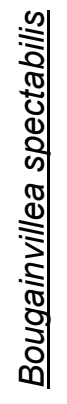 & 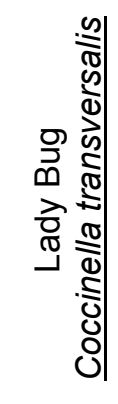 & 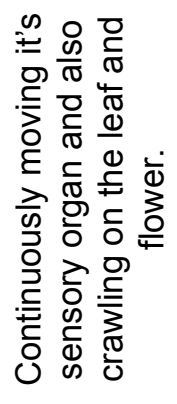 & 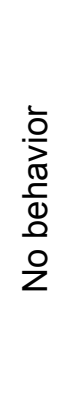 & 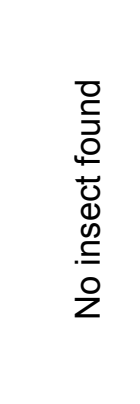 & 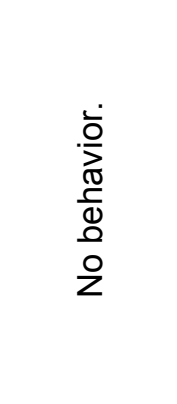 & 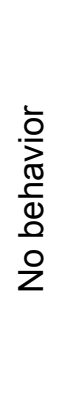 \\
\hline
\end{tabular}




\begin{tabular}{|c|c|c|c|c|c|c|c|}
\hline 22. & 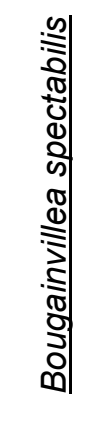 & 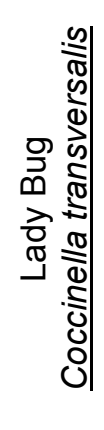 & 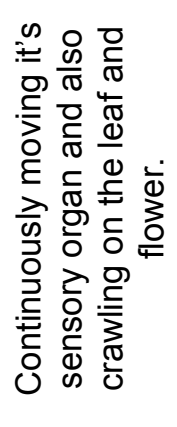 & 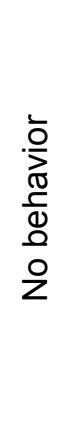 & 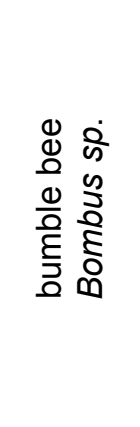 & 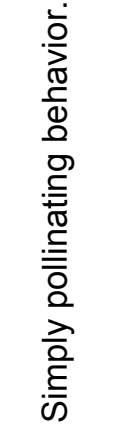 & 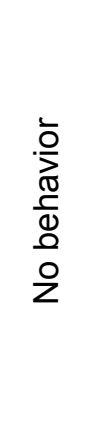 \\
\hline 23. & 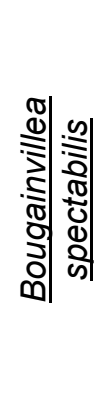 & 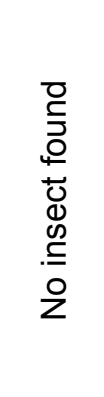 & 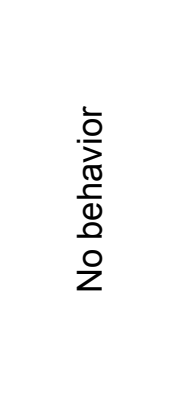 & 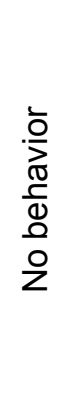 & 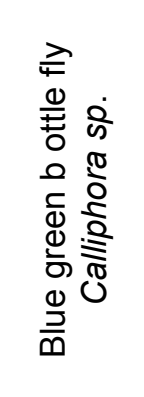 & 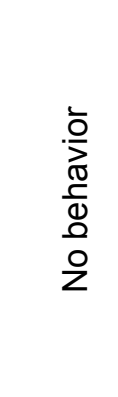 & 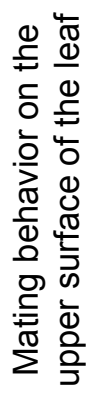 \\
\hline 24. & 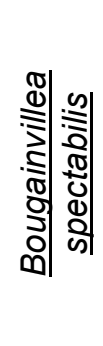 & 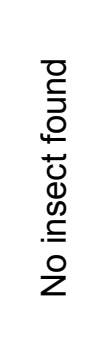 & 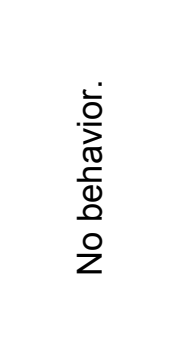 & 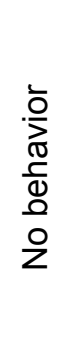 & 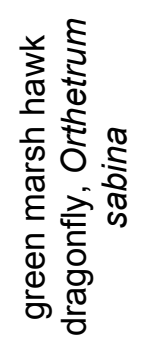 & 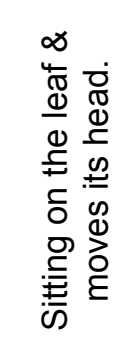 & 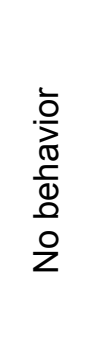 \\
\hline 25. & 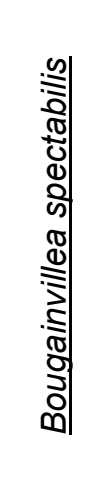 & 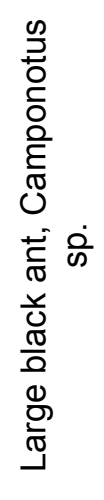 & 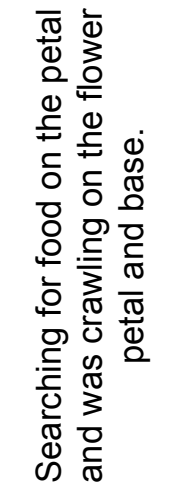 & 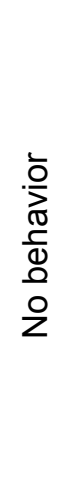 & 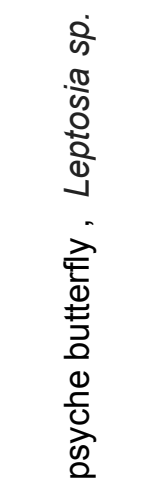 & 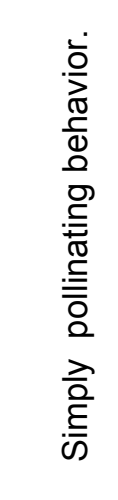 & 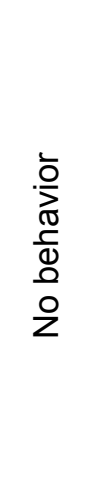 \\
\hline 26. & 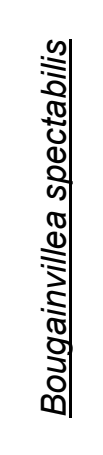 & 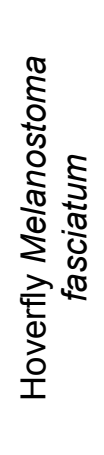 & 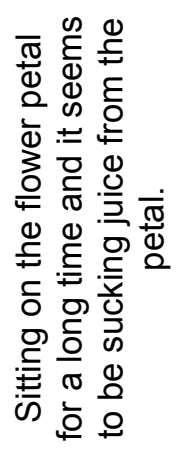 & 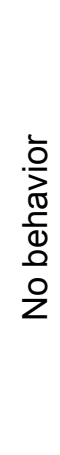 & 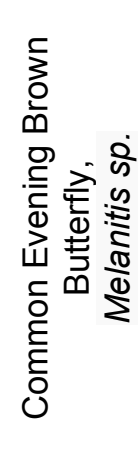 & 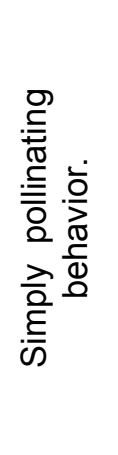 & 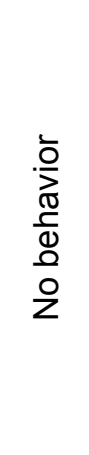 \\
\hline
\end{tabular}




\begin{tabular}{|c|c|c|c|c|c|c|c|}
\hline 27. & 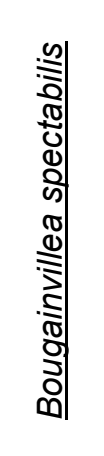 & 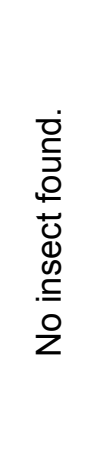 & 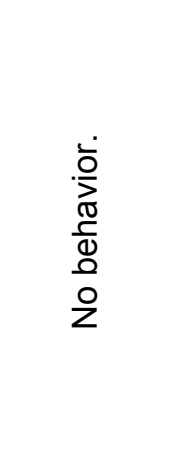 & 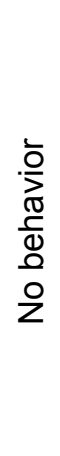 & 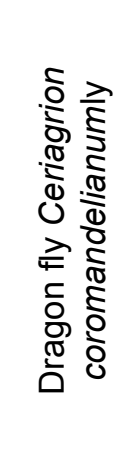 & 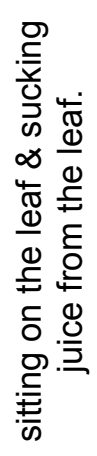 & 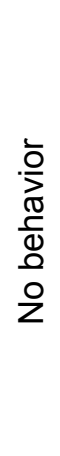 \\
\hline 28. & 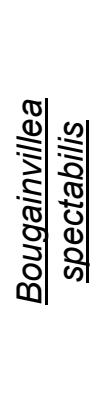 & 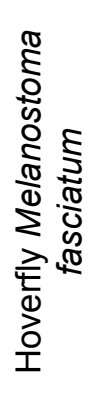 & 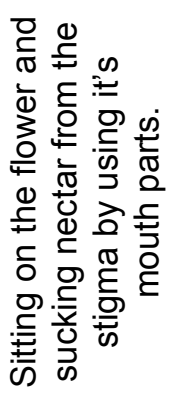 & 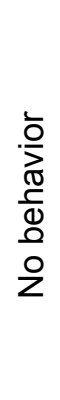 & 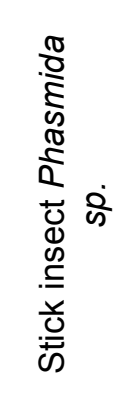 & 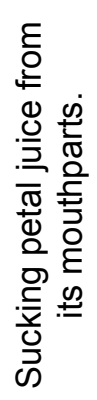 & 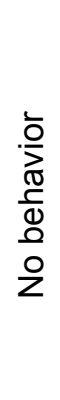 \\
\hline 29. & 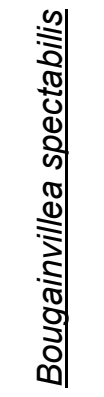 & 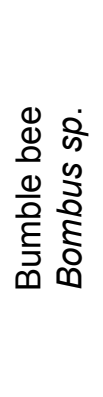 & 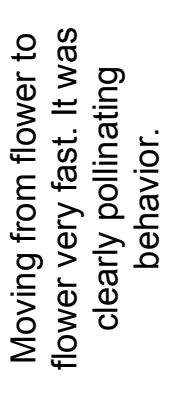 & 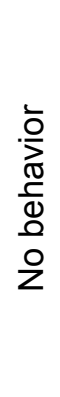 & 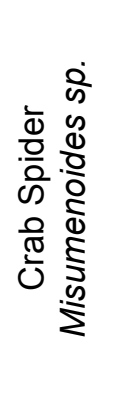 & 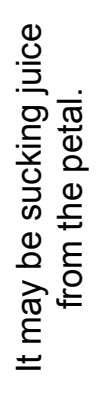 & 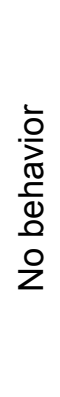 \\
\hline 30. & 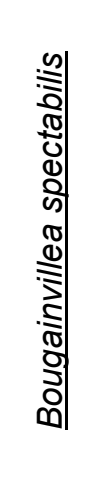 & 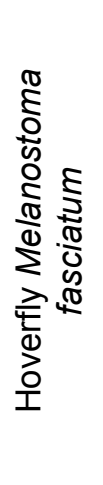 & 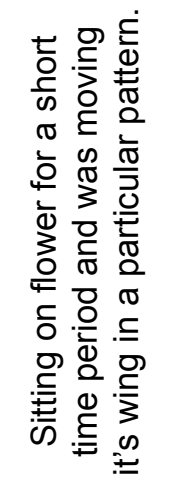 & 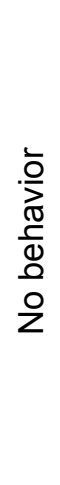 & 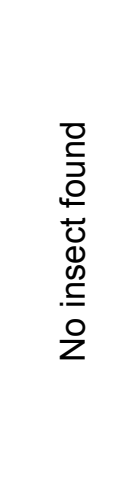 & 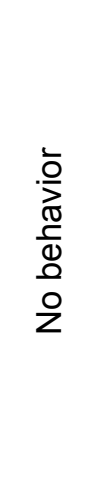 & 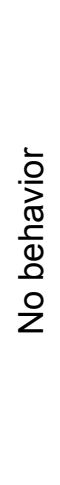 \\
\hline
\end{tabular}

This species was only found in Petunia sp. in Elliot Park while it was present in all three flowering species in Agri-horticulture society. It was mainly showed feeding behavior by sucking juice from flower petal. Lady bug beetle was showed mainly feeding behavior and searching for food but a particular and continuous movement of their sensory organ was observed in Elliot park which was absent in Agri-horticulture society. Honeybees were showed a preference in sunflower as their source of food in both of the park, but there was absent in other flower of Agri-horticulture society while honey bees were showed feeding and searching behavior in petunia in Elliot park. Bumble bee was present in both the parks 
and was showed feeding behavior in the sunflower of Elliot park and it was also showed pollinating behavior clearly in Bougainvillea flower in both parks. Hoverfly was also observed resting for a very long time on the flower and it was seemed to be sucking petal juice. They were present in Elliot Park, while in Agri-horticulture society, only housefly was found but there was no hoverfly. Butterfly was absent in Elliot Park, in comparison with Agri-horticulture society. It was showed clearly pollinating behavior on bougainvillea flower and was also moved its wings in a particular linear direction while sitting on Petunia sp. A crab spider was showed simply feeding and foraging behavior on bougainvillea at Agrihorticulture society. Mating behavior was observed very rare in two managed parks. No insect was showed any kind of mating behavior in Elliot Park, while in Agri-horticulture society two blue/green bottle flies were showed clearly mating phase on the upper surface of the bract of Bougainvillea flower.

\section{DISCUSSION AND CONCLUSION}

The present study of behavioral responses or ethology of flower visiting insects in two managed park namely Elliot Park and Agri-Horticulture Society indicate that there were variable changes among the behavioral responses of same species in two different parks and also the species diversity was more or less different in two parks. The diversity variation has already been studied by Chowdhury et al., (2014).

Generally the green spaces' (e.g. cemeteries, parks, gardens) study have showed an important research area within cities to determine urban ecology. However, study of biotic communities besides office and residential buildings is important because the area, where humans spend most of their time, they interact with nature and where most ecosystem services are likely interacting on a daily basis (Pickett and Cadenasso 2008). The difference shown in two different parks may be vary on certain factors such as human interference, as Elliot park is a public park, vehicular air pollution as Elliot park is just beside the road side and landscape change. Insect outbreaks have been observed in the surroundings of polluted industrial areas and along highways (Holopainen, 2002). According to Grimm et al., (2000), human actions dramatically altered the functioning of ecosystems of which humans are a part, and equally, humans are a part of virtually all ecosystems and have been so for millennia. Nowhere has this human participation been more intense than in cities, suburbs, and exurbs and in the supporting hinterlands. Researchers at the University of Virginia in Charlottesville report that three common constituents of smog destroy floral scents released by flowers to attract bees and other pollinators (Potera, 2008), which may affect the insect behavioral responses. According to McFrederick et al., (2009), chemical signals play important roles in ecological interactions but are vulnerable to perturbation by air pollution. In polluted air masses, signals may travel shorter distances before being destroyed by chemical reactions with pollutants. Pollination, attraction of natural enemies of plant pests, aggregation pheromones, and mate attraction are likely to be affected (McFrederick et al., 2009).

It was observed in the present results (Table 1) that when comparison was made between Elliot park and Agri-horticulture society, various insects viz. small red and black ant, fire ant, dragon fly, lady bug beetle, honey bee, large black ant, bumble bee, hoverfly were observed in Elliot park while in Agri-horticulture society, it was observed some of the above mentioned species alongwith psyche butterfly, leaf beetle, blue/green bottle fly, stick insect and crab spider. 
In this present study it was observed that insects were preferred particular flower for their bright clour, shape, nectar, fragrance, food sources etc. All the honey bees were observed in sunflower and petunia flower and were showed feeding and searching behavior. This was an evidence of other research works (Winston, 1991; Celebrezze and Paton, 2004; Ushimaru et al., 2007; Whelan et al., 2009) that honey bees are good pollinators.

The small red and black ants were mainly showed nectar searching behavior and crawling behavior on the petal. Dragonflies were less in number and were mainly showed feeding behavior by sucking juice from flower petal. Lady bug beetle was showed mainly feeding behavior and searching for food but a particular and continuous movement of their sensory organ. Hoverfly was also observed resting for a very long time on the flower and it was seemed to be sucking petal juice. A crab spider was showed simply feeding and foraging behavior. The stick insect was showed sucking of petal juice as feeding behavior. Similar observations were established of flower visiting insects on the basis of foraging and feeding behavior (Wehner et al., 1983; Bell et al., 1985; Bell, 1990; Alamu et al., 2013).

Butterflies were showed clearly pollinating behavior and also moved its wings in a particular linear direction while sitting on flowering species, the behavior may be an attraction to the partner as mating behavior (Singer, 1982). No insect was showed any kind of mating behavior in Elliot Park, but only in Agri-horticulture society two blue/green bottle flies were showed clearly mating phase on the upper surface of the bract of Bougainvillea flower. The mating behavior is depending upon many environmental factors (Mondor et al., 2004). Pheromones are utilized by insects for several purposes, including alarm signaling (Kislow and Edwards, 1972; Blatt et al., 1998; Hunt et al., 2003), kin recognition (Dani et al., 2001; Vander Meer and Alonso, 2002) and sexual communication (Pickett et al., 1992; Landolt and Phillips, 1997). It was also reported that insects of various groups visit flowers in search of mates (Proctor et al., 1996; Sugiura, 2007) but the present results have documented mating behavior only in bottle flies among all the present insects, which may be due to the affect of atmospheric changes by automobile air pollution (McFrederick et al., 2009) and there may be the chance of vehicular air pollution affect (CPCB, 2009; Citizen's Report, 2011) because these two parks are located nearby roads. In other way, the behavioral differences among the insects of two managed park may be on the size, colour or fragrance of these studied flower.

In the present study it was concluded that the flower visiting insects were observed majorly foraging and feeding behavior while mating behavior was found only two insect species among all other species, which may be the effects of individual and/or combination of automobile air pollutants (McFrederick et al., 2009). It was already reported that Indian cities are susceptible to automobile air pollution (Trivedi et al., 2003; CPCB, 2009; Citizen's report, 2011), though there no attempt has been made on physico-chemical properties of present air pollutants. This study is a preliminary assessment of flower visiting insects and their eco-ethological study. Many studies on flower visiting insects have already been done in greenspace, garden etc. in other parts of globe (Bell, 1990; Matteson et al., 2012, Chowdhury et al., 2014) but less work have documented in eco-ethological aspects in managed parks (Borkent and Schlinger, 2008). In addition, further researches are needed in relation to pollination efficiencies of flower visiting insects in the particular flower and air pollution load by using instruments. It was observed that three flowering species viz. sunflower, petunia and bougainvillea are very common both the parks but insect visitors are showing foraging and feeding behavior but only two species were showed mating behaviour, which may be due to the vehicular air pollution because two parks are located nearby roads and continuous vehicular movements were observed. 


\section{Acknowledgement}

The authors convey their gratitude to the Department of Environmental Science, University of Calcutta, for providing the necessary infrastructure for doing this study.

\section{References}

[1] Alamu O.T., Amao A.O., Oke O.A., Suleiman R.A., Int J Adv Agri Res 1 (2013) 87-91.

[2] Bell W.J., Ann Rev Entomol 35 (1990) 447-67.

[3] Bell W.J., Tortorici C., Roggero R.J., Kipp, L.R., Tobin, T.R., Anim Behav 33 (1985) 436-448.

[4] Bird D.W., O’Connell J.F., J Archaeol Res 14(2) (2006) 143-188.

[5] Blatt S.E., Borden J.H., Pierce H.D.Jr., Gries R., Gries G., J Chem Ecol 24 (1998) 1013-1031.

[6] Borkent C.J., Schlinger E.I., The Canadian Entomologist 140(2) (2008) 250-256.

[7] Dani F.R., Jones G.R., Destri S. et al., Anim Behav 62 (2001) 165-171.

[8] Cane, J.H., Bees' needs challenged by urbanization. - In: Johnson, E. A. and Klemens, M. W. (eds), Nature in fragments: the legacy of sprawl. Columbia Univ. Press, (2005), pp. 109-124.

[9] Celebrezze T., Paton D.C., Austral Ecol 29 (2004) 129-136.

[10] Chowdhury S., Sarkar S., Nandy A., Talapatra S.N., International Letters of Natural Sciences 10 (2014) 58-68.

[11] Citizen's Report, Centre of Science and Environment, (2011) 1-106.

[12] CPCB, Central Pollution Control Board, New Delhi, (2009).

http://www.cpcb.nic.in /bulletin/ del/2009html.

[13] Grimm N.B., Grove J.M., Pickett S.T.A., Redman C.L., BioScience 50 (2000) 571-584.

[14] Hennig E.I., Ghazoul J., Perspectives in Plant Ecology, Evolution and Systematics 13 (2011) 137-150.

[15] Hernandez J.L., Frankie G.W., Thorp R.W., Cities and the Environment 2(1) (2009) 15. http://escholarship.bc.edu/cate/vol2/iss $1 / 3$

[16] Holopainen J.K., Entomologia Experimentalis et Applicata 104 (2002) 137-142.

[17] Hunt G.J., Wood K.V., Guzman-Novoa E., Lee H.D., Rothwell A.P., Bonham, C.C., J Chem Ecol 29(2) (2003) 453-463.

[18] Kislow C.J., Edwards L.J., Nature 235 (1972) 108-109.

[19] Landolt P.J., Phillips T.W., Ann Rev Entomol 42 (1997) 371-391.

[20] Matteson K.C., Grace J.B., Minor, E.S., Oikos (2012) 01-13.

[21] McFrederick Q.S., Fuentes J.D., Roulston T’ai., Kathilankal J.C., Lerdau M., Oecologia 160 (2009) 411-420. 
[22] Mondor E., Tremblay M., Awmack C., Lindroth R.L., Global Change Biology 10 (2004) 1820-1824.

[23] Murvosh C.M., Fye R.L., Labrecque G.C., Ohio J Science 64(4) (1964) 264-271.

[24] Potera C., Environ Health Perspective 116(8) (2008) A334.

[25] Proctor M., Yeo P., Lack A., The natural history of pollination. Timber Press, Portland, OR (1996).

[26] Pickett J.A., Wadhams L.J., Woodcock C.M., Hardie J., Ann Rev Entomol 37 (1992) 67-90.

[27] Pickett S.T.A., Cadenasso M.L., J Ecol 96 (2008) 8-12.

[28] Ram H., Mathur G., Proc Indian Acad Sci (Anim Sci) 93(4) (1984) 359-363.

[29] Sattler S.E., Funnell-Harris D.L., Pedersen J.F., Food Chem 58 (2010) 3611-3616.

[30] Schlinger E.I., Proceedings of the United States National Museum 106 (1956) 359-375.

[31] Singer M.C., Oecologia 52 (1982) 230-235.

[32] Sugiura S., Abe T., Yamaura Y., Makino S., Naturwissenschaften 94 (2007) 703-707.

[33] Trivedi S., Agrawal M., Rajput M., Indian J Air Poll Cont. 3 (2003) 44-53.

[34] Ushimaru A., Watanabe T., Nakata K., American J Bot 94(2) (2007) 249-258.

[35] Vander Meer R.K., Alonso L.E., Behav Ecol Sociobiol 51 (2002) 122-130.

[36] Wehner R., Harkness R.D., Schmid-Hempel P., Foraging strategies in individually searching anls Cataglyphis bicolur (Hymenuptera: Furmicidae). In Information Processing in Animals, ed. M. Lindauer. Vol. I. New York: Gustav Fischer Verlag (1983).

[37] Whelan R.J., Ayre D.J., Beynon F.M., Annals Bot 103 (2009) 1395-1401.

[38] Winston M.L., The Biology of the Honey Bee, Harvard University Press, (1991) pp. 294, ISBN 970674074095.

[39] Wojcik V.A., J Pollin Ecol 4(7) (2011) 48-56. 